\title{
A REAL TIME COGNITIVE RADIO TEST PLATFORM FOR PUBLIC SAFETY PHYSICAL LAYER EXPERIMENTS
}

\author{
Peter J. Green and Desmond P. Taylor \\ Department of Electrical and Computer Engineering \\ Christchurch, New Zealand
}

\begin{abstract}
This paper describes the concept, architecture and development of a real time, cognitive radio testbed for physical layer, interoperability and spectrum sensing experiments. It is based on a reconfigured wireless 4-transmit 4-receiver multiple input and multiple output (MIMO) Smart Antenna Software Radio Test System (SASRATS) platform using the the Xilinx University Program (XUP) Virtex-II Pro development boards as the heart of the system. We discuss various cognitive experiments in the context of public safety applications and show that cyclostationarity can be used for spectrum sensing and recognition applications.
\end{abstract}

\section{INTRODUCTION}

It is reported [1] that at any given time only 14 percent of the radio spectrum is used and this includes huge portions of spectrum that are assigned to TV stations but are not utilised. This under-utilization of the spectrum can be drastically improved through the use of cognitive radios. Cognitive radio is envisioned as the ultimate radio system that can sense, adapt and learn from the environment in which it operates. It is able to sense where and when these spectrum holes [2] exist and to use them appropriately without interference to others. Cognitive radio is software defined radio with the ability to sense the environment and adapts to different frequencies, power levels, protocols, modulation schemes of other radios to enable seamless interoperability while maximising spectrum efficiency.

Other than the obvious military interests in this type of radio, the greatest non-military application of cognitive radios will be in public safety organizations [3]. Public safety organizations such as police, fire and ambulance units can rarely communicate directly to one another without the use of a central dispatcher. Communication chaos after a major disaster such as hurricane Katrina's onslaught on New Orleans in August 2005 have been reported [3].

We present in this paper, a cognitive radio testbed that will enable us conduct real-time physical layer experiments to test and verify algorithms used for spectrum sensing, adaptation and interoperability primarily in the interest of public safety.

\section{EXPERIMENTAL REQUIREMENTS}

Our cognitive radio testbed implementation is an architectural enhancement to an existing $4 \times 4$ MIMO Smart Antenna Software RAdio Test System (SASRATS) platform [4, 5] originally designed to test and verify various space time architectures and algorithms. As the transmit and receive systems of the SASRATS platform are software defined and modular in design, we have changed the architecture to allow the conduct of real time cognitive radio experiments. In the original MIMO architecture, the system exists as separate autonomous transmitter and receiver units. Here we combine the units together in such a way that we have transceivers; both transmitter and receiver coexisting together and use FPGAs and DSPs to perform the signal processing needed for cognitive radio experiments. We refer to the new architecture as the enhanced SASRATS platform or eSASRATS for short.

Experiments we wish to perform on the cognitive eSASRATS platform include

- seamless interoperability between radios having different operating frequencies, modulation and protocols.

- spectrum sensing to determine the presence of primary users and and availability of spectrum holes.

- determination of modulation and multiple access features(type, baud rate) of users

. continuous monitoring (tracking) of spectrum to protect the reappearance of the primary user.

- receiver diversity combining or transmit diversity algorithms to mitigate fading.

. interference cancellation algorithms such as beamforming and beam nulling.

. MIMO cognitive radio algorithms.

\section{A Interoperability}

Interoperability between radios having different operating characteristics is a "must have" feature in a cognitive radio. In a disaster situation following a major earthquake, tsunami, forest fire or flood, first responders need to establish voice and data communication with other first responders. Emergency responders from various districts, jurisdiction or countries will have to communicate effectively to get the rescue effort going. Victims may call for help on their AM Citizens Band radio, cell phones, walkie-talkies (narrowband FM Family Radio Service radios) or even cordless phones but this may be impossible if the corresponding telecomunication infrastructure of the affected area is knocked out. Thus the ability to detect and establish communication interoperability seamlessly is a key feature.

To this effect, we plan to conduct real-time cognitive radio experiments on our testbed to test various detection algorithms and to establish a communication bridge and interoperability between 2 primary users extending to 4 users. We consider both half duplex and full duplex communication links. 
In a half duplex bridge experiment, the cognitive radio bridges 2 radios operating at 2 different frequencies $\left(f_{1}, f_{2}\right)$ and using two different modulations $\left(m_{1}, m_{2}\right)$ and protocols $\left(p_{1}, p_{2}\right)$. In the half duplex mode, each radio transmits and receives using one unique frequency. When one transmits, the other listens. This mode is adopted extensively in walkie talkies used by public safety organizations and also in Citizen Band radios. The cognitive radio bridge must detect when either one of the radios transmits, determine which radio it is, tune in to receive, demodulate the received signal and modulate the transmitter according to the other radios modulation scheme. Appropriate detection, demodulation, modulation, protocol and control algorithms need to implemented and tested. The testbed should also be able to conduct 3 way half duplex voice communication between the cognitive radio testbed and the two radios. This means that the testbed must have capability to simultaneously transmit through 2 transmitters at frequencies $f_{1}$ and $f_{2}$, modulation $m_{1}, m_{2}$ and protocols $p_{1}, p_{2}$ respectively. The testbed should also have the ability to accept another receiver if there is a need to implement diversity combining algorithms to mitigate fading or beamforming to improve reception of a desired signal or beam nulling to cancel out a strong interferer.

The cognitive testbed must also be able to perform full duplex communication interoperability. In full duplex communication, there are separate transmit $\left(f_{1}, f_{2}\right)$ and receive $\left(f_{3}, f_{4}\right)$ channels for communications. Here there is a requirement to link 2 radios where each radio transmits and receives at different frequencies, modulation and protocols to have seamless and simultaneous talk and listen capability. In a possible public safety scenario, disaster victims may try to get help on their cellular or home cordless phones and the ability to scan and receive these full duplex systems is a must. The most basic full duplex testbed will consist of 2 receivers and 2 transmitters controlled by cognitive algorithms. This will enable the testbed to bridge two different radios and also establish full duplex communications with both of them. With additional receivers added-on to the testbed, direction finding algorithms can estimate the direction of arrival of these signals to help rescuers locate them.

\section{B Spectrum sensing}

An essential feature of cognitive radio is its ability to sense the presence of radio signals within a broad spectrum of frequencies. In a public safety scenario, the cognitive radio needs to sense where the spectral holes are and to use them to establish communications between emergency responders. The cognitive radio should also have the ability to determine the frequency, type of modulation and protocol used by emergency responders and also the signals sent out by victims seeking help so that communication and interoperability can be established.

To perform spectrum sensing, several non-coherent detection approaches such as energy detection and cyclostationarity can be used [6]. Energy detection uses the approach used in spectrum analysers and can be implemented in the cognitive baseband processor by averaging frequency bins of a Fast Fourier Transform (FFT). To resolve narrowband signals, the sample size of the FFT must be increased to improve resolution. To detect weaker signals, the signal-to-noise ratio (SNR) can be improved by increasing the averaging time. The drawback to this method is that the energy detected must be compared to some threshold which will determine the probability of detection. This method however cannot easily distinguish between the desired modulated signal, noise or interference and therefore does not work for spread spectrum signals. The FFT energy detector method however is still a good first approach to the spectrum sensing problem as it is a non coherent detector and is relatively simple to implement in DSP or FPGA on our cognitive radio testbed. A more sophiscated method that exploits the cyclostationarity properties of the detected signal provides more information regarding the modulation type, data rate and other spectral signature of the unknown signal is proposed.

Every type of radio transmission especially digital transmissions exhibit a distinct cyclostationary signature that can be exploited to detect and determine their modulation format and baud rate. This occurs because digitally modulated signals have periodicity associated with their baud rate, modulation schemes, training sequences, cyclic prefixes, spreading or hopping sequences. Signals are said to exhibit cyclostationarity if their cyclic autocorrelation or cyclic conjugate correlation functions are nonzero either at some time delay or at some frequency shift. This frequency shift is known as the cyclic frequency $(\alpha)$.

A signal, $x(t)$, has second-order periodicity only if its nonzero cyclic autocorrelation, shown in (1), exists [7], as

$$
R_{x}^{\alpha}(\tau)=\lim _{T \rightarrow \infty} \frac{1}{T} \int_{-T / 2}^{T / 2} x(t+\tau / 2) x^{*}(t-\tau / 2) e^{-j 2 \pi \alpha t} d t
$$

The Fourier transform of the cyclic autocorrelation function is the cyclic spectral correlation density (CSCD) function [8]. In this paper, discrete (sampled) signals are used. The CSCD for a discrete signal is defined in (2) and is a function of both $f$ and $\alpha$, as

$$
S_{x}^{\alpha}(f)=\sum_{-\infty}^{\infty} R_{x}^{\alpha}(\tau) e^{j 2 \pi f t}
$$

For $\alpha=0, S_{x}^{0}(f)$ is just the power spectral density (PSD) of the signal but for non-zero $\alpha$ values, the CSCD is the cross-spectral density of that signal and its frequency shifted version. The CSCD at $\alpha= \pm 1 f_{b}, \pm 2 f_{b}, \pm 3 f_{b}, \ldots$ belongs to the baud rate $\left(f_{b}\right)$ related correlation. The CSCD at $\alpha=0$ or $2 f_{c}$ belong to the carrier frequency $\left(f_{c}\right)$ related correlation. The CSCD function also exists at $\alpha= \pm 2 f_{c} \pm 1 f_{b}, \pm 2 f_{c} \pm 2 f_{b}, \pm 2 f_{c} \pm 3 f_{b}, \ldots$.

Practical implementation of the CSCD method for signal signature detection is shown in Figure1.

It this implementation, the CSCD function is defined [7] as

$$
S_{x}^{\alpha}(f)=\lim _{B \rightarrow 0} \frac{1}{B}\left\langle\left[h_{B}^{f}(t) \otimes u(t)\right]\left[h_{B}^{f}(t) \otimes v(t)\right]^{*}\right\rangle
$$

where $u(t)=x(t) e^{-i \pi \alpha t}, v(t)=x(t) e^{+i \pi \alpha t}, \otimes$ denotes convolution, $h_{B}^{f}(t)$ is the impulse response of bandpass filter with 


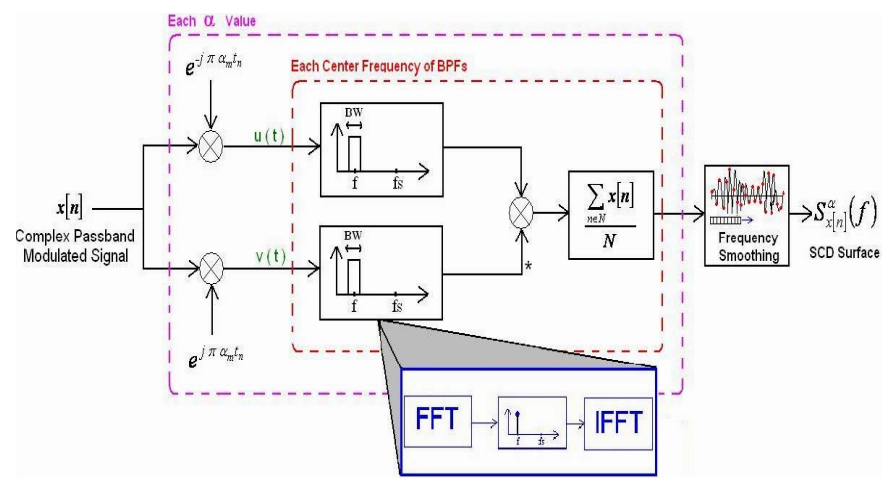

Figure 1: Block diagram of the implementation of the cyclic spectral correlation density function

center frequency $f$ and bandwidth $B$ with unity gain at the band center. $\langle$.$\rangle is a continuous time averaging defined as$ $\lim _{Z \rightarrow \infty} \frac{1}{2 Z} \int_{-Z}^{Z}() d$.

We have investigated the use of the CSCD function for signal signature detection of BPSK, QPSK, OQPSK, MSK and 16QAM modulated signals. We have the CSCD function computed at various values of $f$ and $\alpha$ to produce surface plots of the modulations as shown in Figure 2.

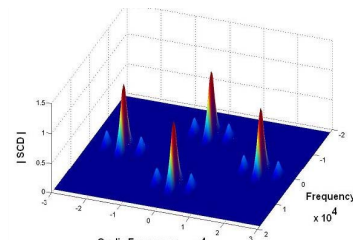

a) SCD of BPSK

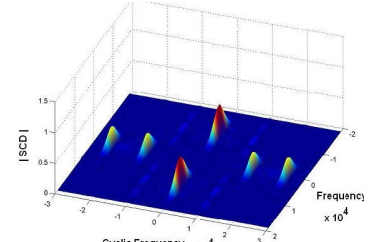

cyclic Frequency $x_{10^{4}}$ SCD $^{2} \mathrm{MSK}$

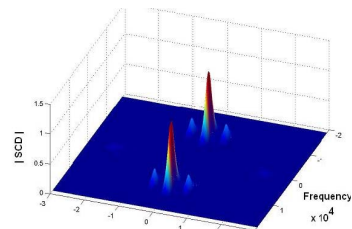

b) SCD of QPSK

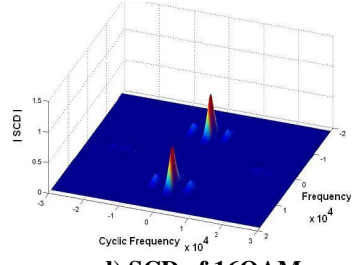

d) SCD of 16QAM
Figure 2: Cyclic spectral correlation density function plots of BPSK, QPSK, MSK and 16QAM signals

Two algorithms were implemented in Matlab for a receiver with apriori knowledge of the carrier frequency and the baud rate of the received signal, and a receiver with no a priori knowledge of either carrier frequency or baud rate. We have found that the various modulation schemes have unique CSCD signature plots. The BPSK and QPSK CSCD plots of Figures $2 \mathrm{a}$ and Figures $2 \mathrm{~b}$ respectively are distinct as they have four and two peaks respectively. However, QPSK and 16QAM (Figure 2d) plots are similar in shape except for amplitude differences between the peaks $\left(f_{c}\right)$ and smaller sidelobes $\left(f_{b}\right)$ and this makes it difficult for the algorithm to distinguish the two as a thresh-
Table 1: Carrier $\left(f_{c}\right)$ and baud rate $\left(f_{b}\right)$ estimation performance and normalised execution times of signal signature detection algorithms using the cyclic spectral correlation density (CSCD) function

\begin{tabular}{l||l|l|l|l|l}
\hline Modulation & \multicolumn{5}{|c}{} \\
\hline Error in $f_{c}(\%)$ & 0.024 & QPSK & OQPSK & MSK & 16QAM \\
\hline Error in $f_{b}(\%)$ & 0.170 & 0.073 & 0.317 & 0.904 & 0.024 \\
\hline Execution Time & & & 1.344 & 3.688 & 0.024 \\
\hline Algorithm 2 & 1 & 3 & & & \\
\hline Algorithm 1 & 0.1 & 0.1 & 0.1 & 0.1 & 0.1 \\
\hline
\end{tabular}

old level must be set. The second algorithm with no apriori knowledge of either carrier frequency or baud rate converges to a solution much more slowly than the first as it has to first estimate $f_{c}$ and $f_{b}$ before proceeding to compare CSCD surface plots. Table 1 shows the performance of the algorithms

The frequency error results in Table 1 are based on comparison with actual values of $f_{c}=10 \mathrm{kHz}, f_{b}=5 \mathrm{Khz}$ at 8 samples per symbol and FFT length of 256. The results show that the CSCD function in the second algorithm can be used to successfully estimate both $f_{c}$ and $f_{b}$ to a high degree of accuracy. However, the error in estimating $f_{b}$ is large if the error in estimating $f_{c}$ is large. This is reflected in highest execution time (in the case of MSK) in recognising the signal signature. The execution times are normalised to the BPSK execution time of algorithm 2. If the carrier frequency and baud rate are known apriori as shown in the last row of Table 1 for algorithm 1 , the execution time is very fast and takes only one-tenth of the normalised standard time. Better frequency error performance can be obtained at the expense of longer FFT length and higher execution time.

These results show that it is possible to use cyclostationarity in cognitive radio applications. However our studies in using the CSCD function motivates us to also study and explore other signal signature detection techniques that are less computationally intensive but offer good performance. We also note that we require a high performance processor in our cognitive radio testbed to perform the FFT's and inverse FFT's operations to implement and test the CSCD function algorithms on the testbed.

\section{OVERVIEW OF THE ESASRATS ARCHITECTURE}

In view of our plans to investigate various aspects of interoperability, spectrum sensing and recognition, diversity and interference mitigation experiments on a real-time cognitive radio testbed, we have developed several options to cater for different levels of capabilities. The architectural options of the cognitive eSASRATS platform are shown in Figures 3 and 4.

At the heart of the cognitive e-SASRATS platform are the Xilinx University Program (XUP) Virtex 2 Pro Development System boards. The versatile XUP board has a Virtex-2 Pro XC2VP30 FPGA with 30,816 logic cells, two PowerPC Processors, 10/100 Ethernet port, USB2 port, Audio Codec, XSGA Video port, SATA(Gigabit serial I/O) port and other high and low speed expansion ports. The PowerPC memory can be expanded to a maximum of 2 Gbytes of RAM. This cost 


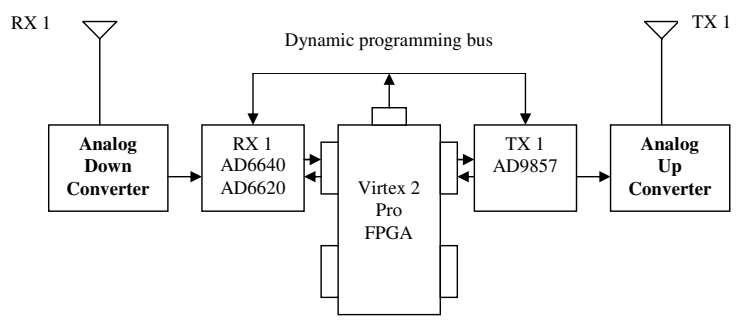

(a)

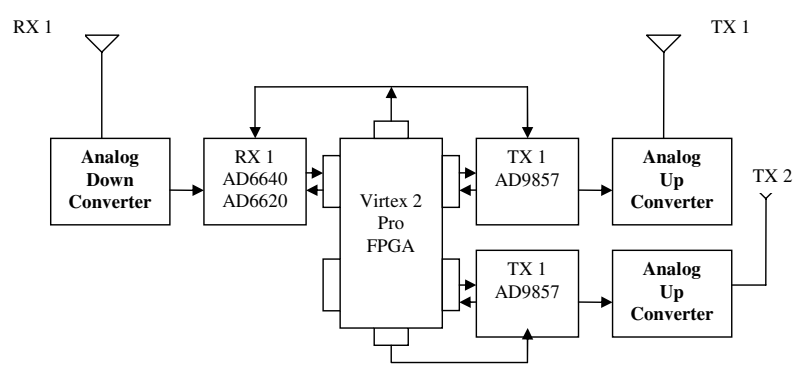

(b)

Figure 3: eSASRATS basic radio system architectures

effective high performance board from Digilent Inc. has four 20-bit wide expansion ports which is ideal to build up a maximum of 2 full-duplex transceiver ( $2 \mathrm{TX} \& 2 \mathrm{RX}$ ) systems. One additional port is available to be used for dynamic programming of the receive downconvertors and the transmit upconvertors. Receiver tuning frequencies, bandwidth, filter characteristics can be dynamically changed including transmitter frequencies, baud rates and other transmit operating characteristics.

The simplest configuration shown in Figure 3a consist of one set of receiver and transmitter units under the control an FPGA to emulate a primary user which if desired, can be remotely controlled via the ethernet. It can also be used as a cognitive radio terminal to bridge two different half duplex radio systems thus permiting 2-way inter-operability. In this mode, the bridge can only listen-in to communication between the 2 radios but cannot talk to both simultaneously. For 3-way half duplex interoperability that enables the cognitive radio bridge, the capability to talk to both radios, will require an additional transmitter to the enabled as shown in Figure 3b. If the system is expanded to include an additional receiver at the fourth available port, then algorithms using diversity combining techniques to mitigate fading or beamforming to mitigate an unwanted interference can be added to improve the quality of service. If a transmitter is connected to the fourth port instead, then it is possible to conduct 4-way half duplex interoperability experiments.

The architecture for full duplex 3-way interoperability between the cognitive radio bridge and 2 primary radios is shown in the top half of Figure 4. In this mode, two sets of transmit and receiver units use all the available ports on the XUP board. The setup can be duplicated such that a second full duplex system shown in the bottom half of Figure 4, can be setup to emulate another communication bridge or merely used to

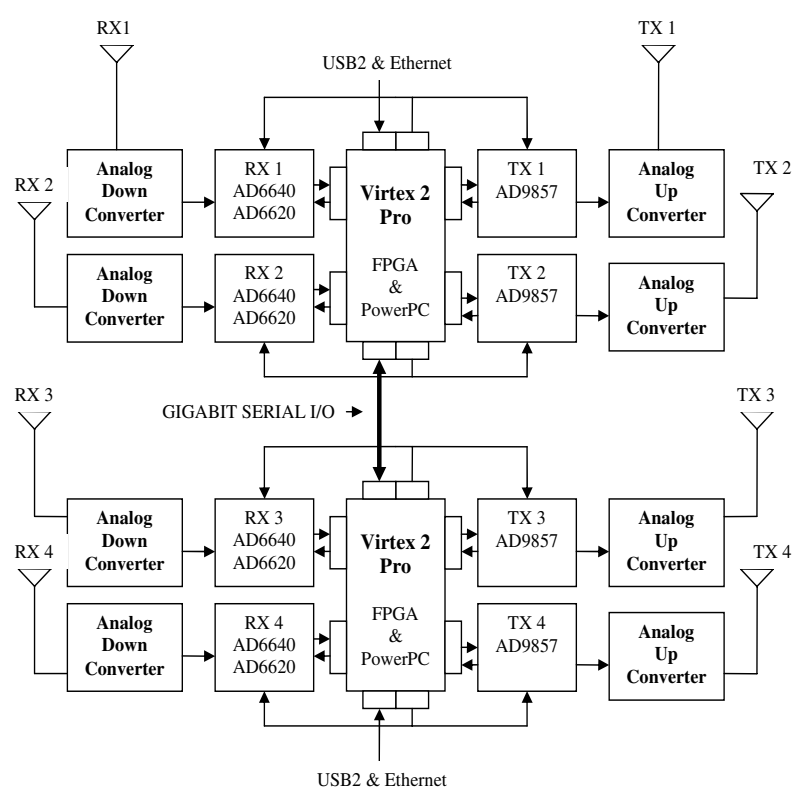

Figure 4: eSASRATS advanced radio system architecture

emulate primary users. The FPGA's of the two systems can be configured to work independently or they can work together by communicating directly using the Rocket I/O Gigabit serial port or remotely via ethernet depending on the nature of the experiment.

The analogue portion of the receiver amplifies, translates and filters a received radio frequency signal at $915 \mathrm{MHz}$ or $2.4 \mathrm{GHz}$ to a received intermediate frequency where digitization and bandpass sampling occurs. The output of the analog to digital converter (AD6640) is then fed into a digital down converter (AD6620) which digitally downconverts, decimates and filters the input data to produce baseband in-phase (I) and quadrature phase $(\mathrm{Q})$ signals for further processing in DSP and FPGA. At the transmitter, I and Q data are fed into a digital upconverter and modulator (AD9857)which outputs a modulated intermediate frequency signal. The analog portion of the transmitter then filters, amplifies, upconverts then power amplifies the transmit signal to $915 \mathrm{MHz}$ or $2.4 \mathrm{GHz}$.

In our testbed, the local oscillator (LO) used in the up and downconversion process is fixed at one frequency which restricts operation in the required Industrial, Scientific and Medical (ISM) band. Our receiver and transmitter analogue convertor design is modular and wideband and it is thus possible to use separate LOs' if desired, to receive and transmit at frequencies outside the ISM band. The receiver is able to receive a large number of channels by digitally tuning to different receive frequencies within the $10 \mathrm{MHz}$ bandwidth of the $70 \mathrm{MHz}$ IF filters. At the transmitters, different transmit frequencies are achieved by programming the digital upconverters to different carrier frequencies within the IF bandwidth. Thus the system has the capability to dynamically receive or transmit under the control of cognitive algorithms.

The various cognitive algorithms on the XUP board will be 
implemented using the Xilinx Integrated System Enviroment (ISE) Foundation suite and also the Xilinx Embedded Development Kit (EDK) with the Platform Studio tool suite. We will also make extensive use of various Xilinx Core Generator intellectual property(IP) modules incorporated within the ISE Foundation toolset to shorten design cycle time. We have used ISE and Core Generator successfully in many real-time MIMO implementations as reported in $[9,10,11]$. To further reduce design cycle time, we intend to reuse transmit and receive firmware and software modules developed in these implementations in the cognitive radio testbed. We also intend to use the Xilinx System Generator for DSP for rapid prototyping. It enables us to develop highly parallel systems in FPGA through system modeling and automatic code generation from Simulink and MATLAB.

\section{CONCLUSIONS}

We have described in this paper, experiments that are important in the evaluation of various cognitive radio operations for public safety applications. We have shown that cyclostationarity and in particular the CSCD function, can be used to estimate the carrier frequency, baud rate and recognise the modulation of an unknown signal. We have described the concept and architecture of a real time cognitive radio test platform for interoperability and spectrum sensing experiments based on the reconfiguration of our current $4 \times 4 \mathrm{MIMO}$ platform with the XUP boards to dynamically control transceiver characteristics whilst performing cognitive algorithms.

\section{$\mathrm{V}$ ACKNOWLEDGEMENT}

We wish to thank Mr. Maung Thant Zin Shein for evaluating the CSCD function, producing the CSCD plots and data used in Table 1.

\section{REFERENCES}

[1] R. Rubenstein, "Radios get smart", IEEE Spectrum, vol. 44, no. 2, pp. 46-50, Feb. 2007

[2] S. Haykin, "Cognitive Radio: Brain-Empowered Wireless Communications", IEEE Journal on Selected Areas in Communications, vol. 23, no. 2, pp. 201-220, Feb. 2005

[3] J. Donovan, "Cognitive Radios solve a host of problems", Portable Design, pp. 8-11, Jan. 2007

[4] P. J. Green and D. P. Taylor, "Smart Antenna Software Radio Test System", Proceedings of the First IEEE International Workshop on Electronic Design, Test and Applications., vol. 1, pp. 68-72, Jan. 2002

[5] P. J. Green and D. P. Taylor, "Experimental verification of space-time algorithms using the smart antenna software radio test system (SASRATS) platform", 15th IEEE International Symposium on Personal, Indoor and Mobile Radio Communications, 2004. PIMRC 2004. , vol. 4, pp. 2539-2544, Sept. 2004

[6] D. Cabric, S. M. Mishra and R. W. Brodersen, "Implementation issues in spectrum sensing for cognitive radios", Signals, Systems and Computers, 2004. Conference Record of the Thirty-Eighth Asilomar Conference on, vol. 1, pp. 772-776, Nov. 2004

[7] W.A. Gardner, Cyclostationarity in communications and signal processing, IEEE Press, 1994

[8] W.A. Gardner, Statistical Spectral Analysis: A Nonprobabilistic Theory, Prentice Hall, 1987
[9] P. J. Green and D. P. Taylor, "Implementation of a High Speed Four Transmitter Space-Time Encoder using Field Programmable Gate Array and Parallel Digital Signal Processors", Proceedings of the Third IEEE International Workshop on Electronic Design, Test and Applications, pg. 466-471, Jan. 2006

[10] P. J. Green and D. P. Taylor, "Implementation of Four Real-Time Software Defined Receivers and a Space-Time Decoder using Xilinx Virtex 2 Pro Field Programmable Gate Array", Proceedings of the Third IEEE International Workshop on Electronic Design, Test and Applications, pg. 89-92, Jan. 2006

[11] P. J. Green and D. P. Taylor, "Implementation of a Real-Time Multiple Input Multiple Output Channel Estimator on the Smart Antenna Software Radio Test System Platform using the Xilinx Virtex 2 Pro Field Programmable Gate Array", "Proceedings of the 2006 IEEE International Conference on Field Programmable Technology, pg. 257-250, Dec. 2006 